\title{
A General Template to Configure Multi-Criteria Problems in Ubiquitous GDSS
}

\author{
João Carneiro $^{1}$, Diogo Martinho ${ }^{1}$, Goreti Marreiros ${ }^{1}$ and Paulo Novais ${ }^{2}$ \\ ${ }^{1}$ GECAD - Knowledge Engineering and Decision Support Group, Institute of Engineering - \\ Polytechnic of Porto, Porto, Portugal \\ \{jomrc, 1090557, mgt\} @isep.ipp.pt \\ ${ }^{2}$ CCTC - Computer Science and Technology Center, at University of Minho, \\ Braga, Portugal \\ pjon@di.uminho.pt
}

\begin{abstract}
The study of multi-criteria problems adapted to the context of Ubiquitous Group Decision Support Systems (UbiGDSS) is covered in the literature through very different perspectives and interests. There are scientific studies related to the multi-criteria problems that lie across argumentation-based negotiation, multi-agent systems, dialogues, etc. However, to perform most of these studies, a high amount of information is required. The usage of so much data or information that is difficult to collect or configure can bring good results in theoretical scenarios but can be impossible to use in the real world. In order to overcome these issues, we present in this paper a general template to configure multi-criteria problems adapted for the contexts of UbiGDSS that intends to be easy and fast to configure, appellative, intuitive, permits to collect a lot of data and helps the decision-maker transmitting his beliefs and opinions to the system. Our proposal includes three sections: Problem Data, Personal Configuration and Problem Configuration. We have developed a prototype with our template with the purpose to simulate the configuration of a multi-criteria problem. We invited real decision-makers to use our prototype in a simulated scenario and asked to them to fulfil a survey in the end in order to study our hypotheses. Our general template achieved good results and proved to be very perceptible and fast to configure.
\end{abstract}

Keywords: Ubiquitous Group Decision Support Systems, Multi-Criteria Problems, Affective Computing, Multi-Agent Systems, Problem Definition.

\section{$1 \quad$ Introduction}

Ubiquitous computing has become a necessity while developing Group Decision Support Systems (GDSS) [1, 2]. The problem of group decision-making brought new issues such as how to effectively improve the interaction between all the participants involved in the decision-making process and at the same time "deal with uncertainty, ambiguous problem definitions, and rapidly changing information" [3]. Ubiquitous computing provides answers to these issues by improving the way information flows 
through all the distributed environments [4] and how it allows every participant to exchange knowledge regardless of time or location constraints [1]. However, many existing Ubiquitous Group Decision Support Systems (UbiGDSS) seem to forget about these principles and force the user with either overly complex and slow configurations [1] which no decision-maker with a tight schedule will bother to fill accurately or they do not convey the decision-maker opinion properly into the system resulting in a loss of valuable intelligence [5].

In this work, we propose a general template composed by set of points which we think that should be considered when modelling multi-criteria problems, and that will ultimately allow the system to take advantage of more intelligent mechanisms by dealing with all the information (both subjective and objective) that affects the decision-making process. Besides that, we also think that these points are fundamental to obtain a huge amount of information, not only directly (through their configuration) but also indirectly by how they relate to each other and how they affect the decisionmaking process that will lead to more intelligent and closer to real decisions. We then propose a general template that provides the decision-maker with a simple and perceptible configuration, which will allow him to understand the problem more quickly and at the end will enhance the usability of any system that follows this template. We also developed a prototype of a simple UbiGDSS (user interface) including a specification of our template, in order to perform simulations with real decision-makers. The simulations consisted in the configuration of a simulated scenario, followed by filling out a survey.

The rest of the paper is organized as follows: in the next section is presented our methods, where we: identify three different sections that are relevant to our context; present each one of the sections, describing every point and correlating it with the proposed template. In the section 3, we present the evaluation of our model through the case study that was performed and the results that were obtained. In the following section, we discuss the obtained results in the previous section. Finally, some conclusions are taken in section 5, along with the work to be done hereafter.

\section{General Template}

Defining a configuration environment in which the decision-makers could model a multi-criteria problem is a complex process. Whenever it is planned and designed something with the sole purpose to be used by the type of end users considered in this work (executives and top managers), there should be considered some necessary factors for the UbiGDSS to succeed: non-mandatory configurations, usability, problem data always accessible, allow the decision-maker to model its opinion truthfully, and be complex enough whenever it is necessary.

We have grouped the necessities and the several points that should be presented in a configuration template in three sections: Problem Data, Personal Configuration and Problem Configuration (The Fig. 1 represents the template's structure in the correct order). The Problem Data should always be available and accessible throughout each 
configuration step in order to allow the decision-maker to consult any necessary information as much as he needs.

\begin{tabular}{|ll|}
\hline Section 1 & Problem Data \\
& \\
\hline Section 2 & Personal Configuration \\
& \\
\hline Section 3 & Problem Configuration \\
& \\
\hline
\end{tabular}

Fig. 1. Structure of the proposed general template

\subsection{Problem Data}

In this section, it is presented the problem specific information that is a result of several brainstorming sessions. This information has to be organized, cleaned and structured before the decision-maker performs the problem configuration. In its composition, a problem includes a number of criteria as well as a number of alternatives which are different instantiations of all the considered criteria. Both criteria and alternatives are topics to be discussed by decision-makers and are essential to try to reach a decision to solve the problem. Besides alternatives and criteria it should also be inserted all the information that is relevant to reflect about the problem, such as: historical data, financial and cultural issues, etc.

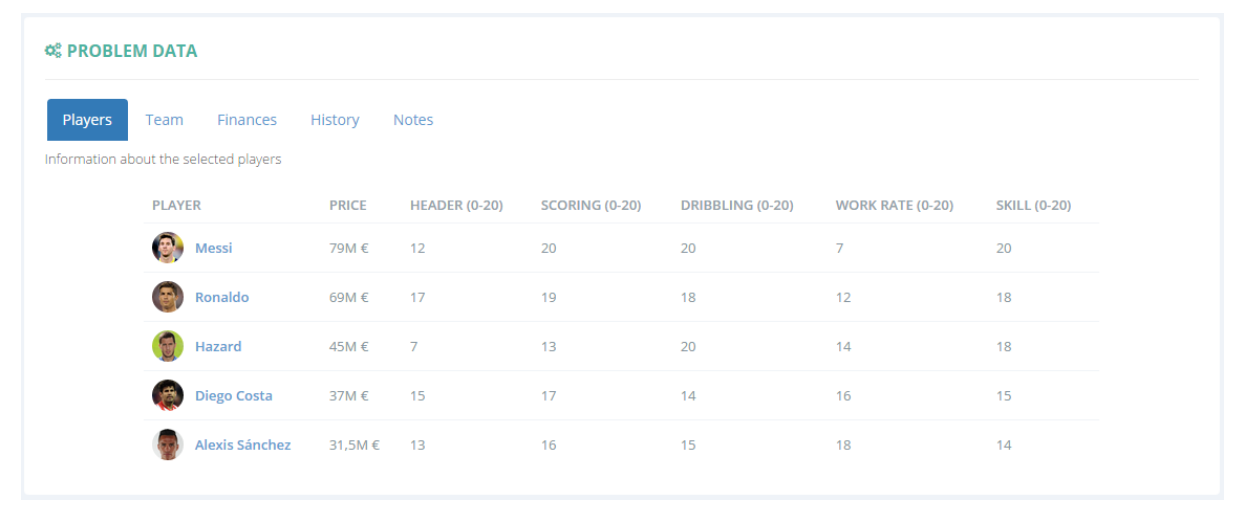

Fig. 2. Problem Data

In our example (Fig. 2) we deal with a scenario of a football technical team that has to decide about which football player (from a range of five options) that should be 
acquired at the start of the next transfer market. Our Problem Data includes all the information about: each one of the five players, the current football team data (which player plays in the current team, statistics, etc.), financial issues, historical data, and other important notes about the problem. All the information is organized and can be consulted by the decision-maker in a very clear and accessible way.

\subsection{Personal Configuration}

This section is related with how the decision-maker can model its own personal attributes as well as his opinion about other decision-makers' attributes. In most of automatic negotiation models, the decision-maker's opinion is considered throughout the entire process in the same way. This means that for those models, the opinion from a decision-maker who does not hold any sort of knowledge towards a topic and still makes a problem configuration will have the same weight as the opinion of an expert in that matter. Another example is the level of interest shown by the decisionmaker in the topic that is not considered. In most of the models it is impossible to know the interest or the commitment level that a decision-maker plans to bring to the decision-making process. Because of these factors, most models are not able to properly generate intelligence, and end up generating garbage instead of valuable information.

The points that we propose to be modelled in Personal Configuration will allow a much easier and intuitive configuration, never forgetting that none of them are mandatory. The proposed points are: Expectations, Expertise Level, Conflict Style, Credibility and Notes. As can be seen in Fig. 3, this type of configuration can be easily done using a mouse, keyboard or a touchscreen, and should take no longer than 2 minutes. Besides that, these points will allow to obtain a huge amount of direct and indirect information.

Expectations. The inclusion of the decision makers' expectations can raise some questions in the literature because the majority of the current negotiation models do not mention this issue. However, there are a few works which are starting to consider the expectations in its analysis $[6,7]$. Evaluating the expectations of the decisionmaker will allow creating a richer negotiation process, which will lead to a better understanding and representation of the decision-maker [8]. This evaluation can also be used, as shown in some recent scientific researches in the calculus of the decisionmaker's satisfaction.

Expertise Level. Expertise is considered as one of the credibility dimensions (affected by objective components) [9]. Our objective in this point is to allow the decisionmaker to make a self-evaluation about his expertise level for the topic at hand. We have considered the existence of five different expertise levels: Expert, High, Medium, Low and Null. Why is this information relevant and how can it be used? This kind of information can have many applications. One could be to use it to compare the self-evaluation made by the decision-maker for its expertise level with its credibil- 
ity which is recognized by other decision-makers, allowing further conclusions to be made. This information can also be used to compare the self-evaluation with the chosen conflict style.

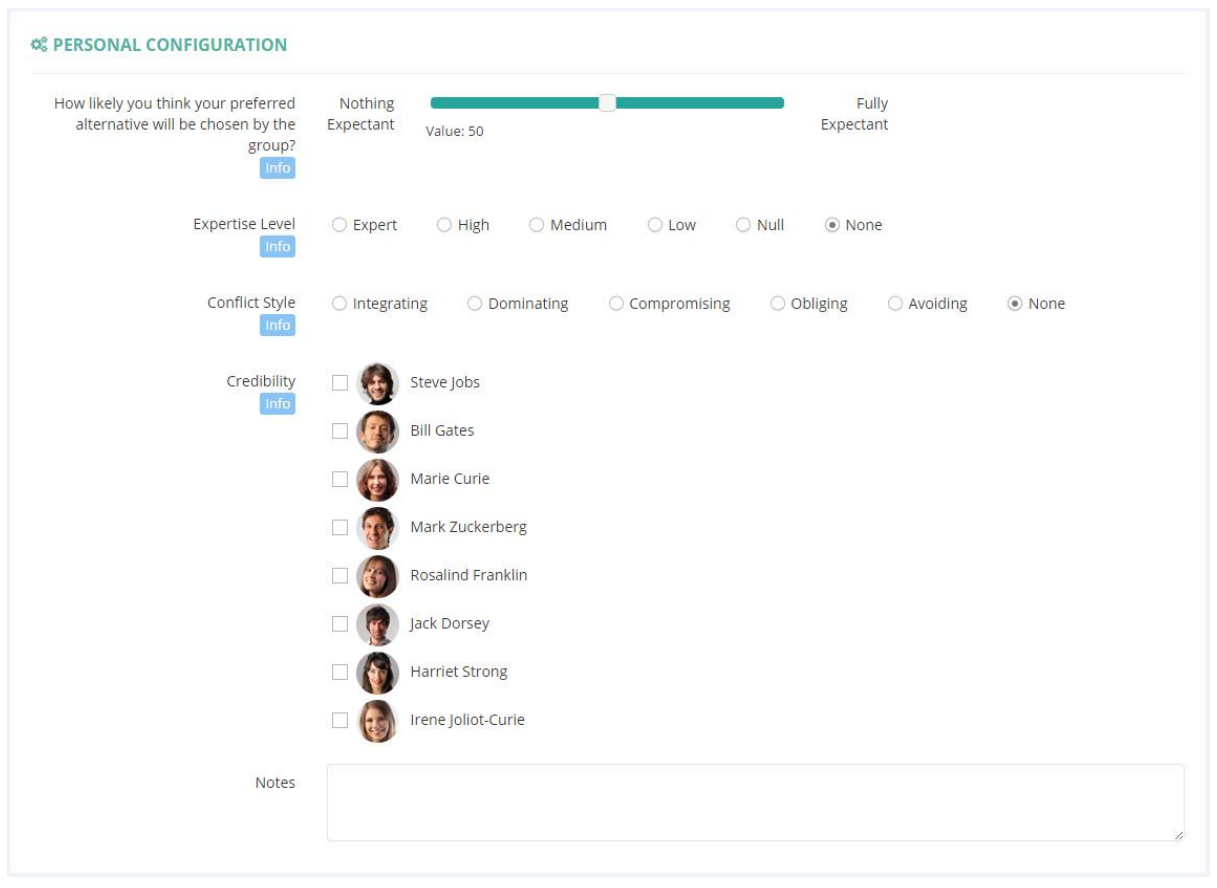

Fig. 3. Personal Configuration

Conflict Style. The conflict style can also be seen as the expected behaviour or the desirable behaviour. In some of our previous works we studied the difference between using the decision-maker's personality or to create the possibility for the decisionmaker to select the conflict style or the behaviour that he intends to use (to read more about this see [10]). During our life, we are constantly presented with the need to take decisions. Some decisions are less relevant, others are not. Fundamentally, when we are taking decisions in group, and independently of our personality, we express and behave differently according to different situations such as: the topic of the problem to be solved, to consider the others decision-makers more expert than us, to intend to be pleasant and try to pursue the others' goals, the level of the interest on the topic, the mood, etc. This means that in a hypothetical scenario where I and my family are deciding from a group of alternatives a restaurant to go celebrate my son's birthday, maybe my main concern is to satisfy my son's preferred alternative. However, I still have my own preferences regardless of my kind of behaviour during the negotiation process. 
For this proposal we have followed our previous studies [10] where we identified 5 conflict styles: Dominating, Integrating, Compromising, Obliging and Avoiding. These styles differentiate from each other by what we think that are 4 essential dimensions for this context:

- Concern for Self - This dimension is related to the individual's concern for his own opinion above the others since he is likely to adapt a more one-sided attitude during the decision-making process by making statements, questions and requests that detail that opinion;

- Concern for others - This dimension relates to the individual's concern for other individuals' opinion. He adapts a more altruist attitude during the decision-making process, trying to understand other opinions and making an effort to reach a decision that benefits or pleases most of the participants;

- Activity - This dimension relates to the effort put into the decision-making process by the individual, meaning that the more active an individual is, the more questions and statements and requests he is likely to make;

- Resistance to change - This dimension relates to how hard or easy it is for an individual to accept other opinions.

Table 1. Conflict styles and corresponding dimensions, adapted from [10]

\begin{tabular}{lllll}
\hline Conflict Style & $\begin{array}{c}\text { Concern for } \\
\text { Self }\end{array}$ & $\begin{array}{c}\text { Concern for } \\
\text { Others }\end{array}$ & \multicolumn{1}{c}{ Activity } & $\begin{array}{c}\text { Resistance to } \\
\text { Change }\end{array}$ \\
\hline Dominating & High & Low & High & High \\
Integrating & High & High & High & High \\
Compromising & Moderate & Moderate & Moderate & Moderate \\
Obliging & Low & High & Low & Low \\
Avoiding & Low & Low & Low & Low \\
\hline
\end{tabular}

In Table 1, we describe each conflict style by providing a value for every dimension mentioned before.

Credibility. There is not a universally accepted definition for credibility. Besides that, the study of credibility is highly multi-disciplinary and some of the suggested definitions are related to their area of operation [9]. In our work, we consider the definition proposed by Flanegin and Metzger when they say that "the overarching view is that credibility is the believability of a source or message, which is made up of two primary dimensions: trustworthiness and expertise" [9]. Trustworthiness is related with subjective components while expertise is related with more objective components. The notion of credibility is related with many other concepts including trust, reliability, accuracy, quality, authority, reputation, competence, etc [9]. In our work the decision-maker can select which other decision-makers he considers to be credible towards a certain topic. This credibility evaluation is related with the concepts mentioned above and will be the reason why a decision-maker may consider another decision-maker to be credible for a topic and not for a different topic (for example, with 
the related expertise level recognized for that decision-maker), and also why a decision-maker may consider another decision-maker always credible despite of the topic's difference (for example, due to reasons such as authority, reputation, etc.).

Notes. We have considered important to include something that allows the decisionmaker to express openly his opinion towards its Personal Configuration. Notes can also be important for the systems with natural language mechanisms, to be used as arguments and to understand different situations in the future.

\subsection{Problem Configuration}

This section is related with how problem-specific attributes are modelled. The decision-maker may select: the preference chosen towards each one of the available alternatives; the importance given for each criterion; preferred alternatives and criteria; the preferred alternatives that he can give up on pursuing; alternatives and criteria without opinion, alternatives and criteria with private opinion; and finally notes. In this section, the configuration can also be done very quickly and intuitively. Besides that, it allows to specify the opinion unconsciously according to natural comparison that exists between the given alternatives, which is done by the human being (for more information, see [6]).

Alternatives Classification. The method to classify alternatives, as can be seen in Fig. 4, has been adapted from the Visual Analogue Scale (VAS) because we think that this scale is the most appropriate for our problem. This scale is appropriate for values that cannot be directly measured and that way allow the decision-maker to level his opinion in a range of values instead of writing down a specific value. Besides that, many studies have proven that VAS allows obtaining information more quickly and assertively [11]. In our context it is also important to refer that this facilitates the configuration, improving how the system could be used in many electronic devices. Another advantage from this model is that it improves the way the decisionmaker evaluates all the suggested alternatives and its comparison since, as he starts selecting new values for each alternative, he will inevitably look at what he selected before and also judge his opinion according to that. Because of this we believe that the decision-maker will find the criterion preference configuration process perceptible and easy to use.

Criteria Classification. The same process was applied to the criteria classification where the decision-maker can scale his opinion for each criterion, due to the reasons mentioned before.

Preferred. Decision-maker can select which alternatives are his preferred ones. Assuming this task is easy and fast to complete, it is better that the decision-maker selects his preferred alternatives instead of running an algorithm to create the group of 
preferred alternatives based in the "Alternatives Classification". Besides, this approach permits to the decision-maker to classify all the alternatives negatively in the "Alternatives Classification" and to select his preferred alternatives considering the possible ones.

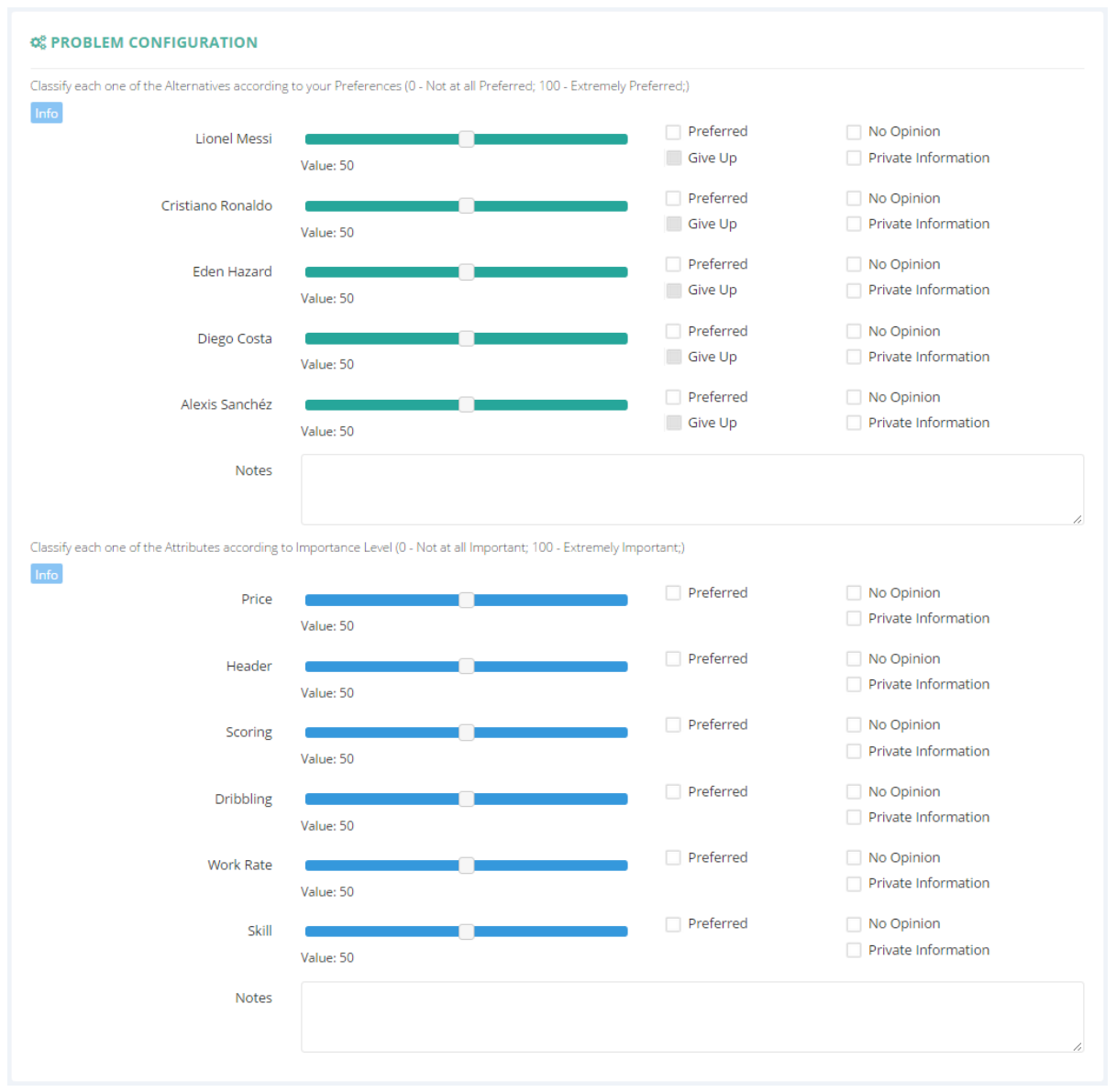

Fig. 4. Problem Configuration

Give Up. The "Give Up" option permits to select the alternatives that for some reason the decision-maker stops to pursue. This point makes sense because in situations when for instance all others decision-makers do not prefer a certain alternative $\mathrm{X}$, does not make sense to still pursue this alternative $\mathrm{X}$, so the decision-maker can indicates to his agent to stop pursue the alternative $\mathrm{X}$. In the past, when the decisionmaker wanted to give this information to the agent, he had to change the classification of the alternative $\mathrm{X}$ in the "Alternatives Classification" (reducing it), but did not make sense to change the classification if the decision-maker did not change the opinion 
about the alternative $\mathrm{X}$, and so it is more intelligent and useful to maintain the same classification and select the "Give Up" option.

No Opinion. Looking at Fig. 4 it is possible to verify that the decision-maker can select the "No Opinion" option for any alternative or criterion. This means that even if the decision-maker wants, for example, to configure the entire problem and if he does not have a formulated opinion for a criterion or an alternative, he is not forced to invent just to configure everything. This can result in relevant information about how the agent should act on behalf of the decision-maker

Private Information. Similarly, the decision-maker may opt to select the opinion about a given alternative or criterion as "Private" (Fig. 4) whenever he intends to not share that knowledge. This may happen, for instance, due to strategical reasons. This information will also be relevant to define how the agent should act.

Notes. The purpose of this point is exactly the same as the notes described in the section of Personal Configuration.

\section{$3 \quad$ Evaluation and Results}

In order to validate our proposal, we conducted a case study which consisted in to ask to random people to fill our template. The only constraint was that the selected people needed to make group decisions regularly in their daily jobs. The study consisted in a first stage to fill a small questionnaire, then they filled out our template and in the end they answered to a small survey. This case study intends to validate our proposal mainly relatively to 3 topics: usability, configuration speed and overall satisfaction.

To perform a better evaluation, we decided to follow a heuristic evaluation. This evaluation was carried out following the 10 Nielsen's Heuristics [12].

\subsection{Case Study}

The performed usability tests were always controlled by three evaluators, in a controlled environment, and always in the same conditions. One of the evaluators was in charge of controlling the time the users took to complete each task, the other observed the errors made, and the last one registered the data obtained and if the decisionmaker pressed the "info" button or not.

\subsection{The Tests}

Besides the configuration of the multi-criteria problem, a questionnaire was conducted, composed of a pre-test to characterize the users (age, gender, profession and work field), and a survey to collect the general perception of the system to the users. 
Questionnaire: pre-test. The users surveyed (30) had their ages between 21 and 55 years old, with an average of 36,5, a standard deviation of 10,09, a mode of 28 and a median of 35 years old. Most of them (57\%) were male users. The Fig. 5 shows the users' age distribution and the frequency of each case.

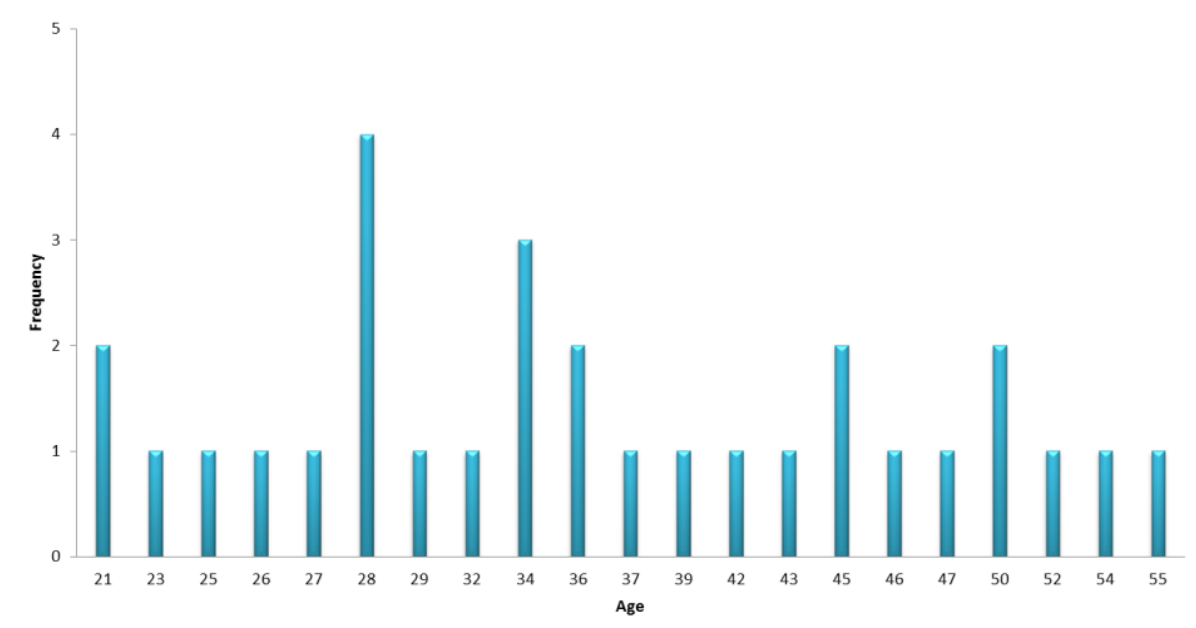

Fig. 5. Users' age distribution

The users answering this questionnaire had different professions like: doctors, economists, engineers, programmers, etc. The majority of the occupations seen were professors and economists. The Table 2 shows the distribution regarding the profession for each one of the users.

Table 2. Users' profession distribution

\begin{tabular}{|c|c|c|c|c|}
\hline Occupation & Frequency & Percent & Valid Percent & $\begin{array}{c}\text { Cumulative } \\
\text { Percent }\end{array}$ \\
\hline Architect & 2 & $6,67 \%$ & $6,67 \%$ & $6,67 \%$ \\
\hline Designer & 1 & $3,33 \%$ & $3,33 \%$ & $10,00 \%$ \\
\hline Doctor & 2 & $6,67 \%$ & $6,67 \%$ & $16,67 \%$ \\
\hline Economist & 5 & $16,67 \%$ & $16,67 \%$ & $33,33 \%$ \\
\hline Engineer & 3 & $10,00 \%$ & $10,00 \%$ & $43,33 \%$ \\
\hline $\begin{array}{l}\text { Human } \\
\text { sources }\end{array}$ & 2 & $6,67 \%$ & $6,67 \%$ & $50,00 \%$ \\
\hline Nurse & 2 & $6,67 \%$ & $6,67 \%$ & $56,67 \%$ \\
\hline Professor & 7 & $23,33 \%$ & $23,33 \%$ & $80,00 \%$ \\
\hline Programmer & 3 & $10,00 \%$ & $10,00 \%$ & $90,00 \%$ \\
\hline Scientist & 3 & $10,00 \%$ & $10,00 \%$ & $100,00 \%$ \\
\hline Total & 30 & $100,00 \%$ & $100,00 \%$ & \\
\hline
\end{tabular}


The users were also from a varied range of work fields: academics, industry, medical, science and technology. However, almost an half of them were from industry (see Table 3).

Table 3. Users' area distribution

\begin{tabular}{lllll}
\hline \multicolumn{1}{c}{ Field } & Frequency & Percent & Valid Percent & $\begin{array}{c}\text { Cumulative } \\
\text { Percent }\end{array}$ \\
\hline Academics & 6 & $20,00 \%$ & $20,00 \%$ & $20,00 \%$ \\
Industry & 12 & $40,00 \%$ & $40,00 \%$ & $60,00 \%$ \\
Medical & 4 & $13,33 \%$ & $13,33 \%$ & $73,33 \%$ \\
Science & 3 & $10,00 \%$ & $10,00 \%$ & $83,33 \%$ \\
Technology & 5 & $16,67 \%$ & $16,67 \%$ & $100,00 \%$ \\
Total & 30 & $100,00 \%$ & $100,00 \%$ & \\
\hline
\end{tabular}

Usability Tests. The usability test consisted only in one task: "perform the configuration of the multi-criteria problem". Considering that all the 3 sections of our template are located in same webpage, we do not have the need to define more than one task. Each decision-maker was put in front of a laptop already with the template open. The configuration was considered to be complete when the decision-maker pressed the button "Submit". None of the configuration fields was/is mandatory. The only 2 things we asked to the decision-makers was (first) to imagine that they were in front of a real scenario and consequently to use their imagination in the configuration of the expectations and the credibility and (second) to complete the configuration in descending order (if they did not want to respond to any point they should express so orally).

Table 4. Number of answers and errors of each point

\begin{tabular}{lllll}
\hline \multicolumn{1}{c}{ Point } & Number of answers & Percent & Number of errors & Percent \\
\hline Expectations & 30 & $100,00 \%$ & 0 & $0,00 \%$ \\
Expertise Level & 30 & $100,00 \%$ & 0 & $0,00 \%$ \\
Conflict Style & 30 & $100,00 \%$ & 0 & $0,00 \%$ \\
Credibility & 13 & $43,33 \%$ & 0 & $0,00 \%$ \\
PC Notes & 0 & $0,00 \%$ & 0 & $0,00 \%$ \\
Alternatives & 30 & 100,00 & 0 & $0,00 \%$ \\
Alt. Notes & 0 & $0,00 \%$ & 0 & $0,00 \%$ \\
Attributes & 30 & $100,00 \%$ & 0 & $0,00 \%$ \\
Att. Notes & 0 & $0,00 \%$ & 0 & $0,00 \%$ \\
Total & 163 & - & 0 & - \\
\hline
\end{tabular}

Our usability test evaluated specifically 3 points: (1) if the decision-makers assess correctly the point that they want to assess; that means, if they are configuring "conflict style" they should not use the evaluation scale of the expertise level to respond (for instance); (2) if they use the "info" button when they need it and (3) how much 
time they need to configure each point. Relatively to the point 1 of the usability test, we witnessed to all the configurations were completed without any errors. To fully complete the configuration the decision-makers needed to answer to 9 points ( 9 points $* 30$ decision-makers $=270$ points). Of the 270 possible points, the decision-makers only answered to 163 points. The main factor to this was that none decision-maker wrote anything in "personal configuration notes", "alternatives notes" and "attributes notes", which resulted in 90 less points. The Table 4 shows the number of errors for each point and the number of decision-makers that answered to each point. Relatively to the point 2 of the usability test, we did not have any users which first clicked in the incorrect "info" button. However it was interesting to analyse the more clicked "info" buttons. The Table 5 shows the number of clicks in each "info" buttons and the number of participants which used each "info" button.

Table 5. Usage level of "info" button

\begin{tabular}{llll}
\hline \multicolumn{1}{c}{ Point } & Number of clicks & $\begin{array}{c}\text { Number of partic- } \\
\text { ipants }\end{array}$ & Percent \\
\hline Expectations & 18 & 15 & $50,00 \%$ \\
Expertise Level & 13 & 12 & $40,00 \%$ \\
Conflict Style & 37 & 30 & $100,00 \%$ \\
Credibility & 24 & 22 & $73,33 \%$ \\
PC Notes & 9 & 9 & $30,00 \%$ \\
Alternatives & 23 & 21 & $70,00 \%$ \\
Alt. Notes & 6 & 6 & $20,00 \%$ \\
Attributes & 16 & 16 & $53,33 \%$ \\
Att. Notes & 4 & 4 & $13,33 \%$ \\
Total & 150 & 135 & - \\
\hline
\end{tabular}

During the test (like we stated before) we controlled the time each decision-maker needed to complete the configuration of each point.

Table 6. Configuration times

\begin{tabular}{llll}
\hline $\begin{array}{c}\text { Configuration } \\
\text { Step }\end{array}$ & \multicolumn{1}{c}{ Average (sec.) } & \multicolumn{1}{c}{$\begin{array}{c}\text { Std. Deviation } \\
\text { (sec.) }\end{array}$} & Median (sec.) \\
\hline C1 & 7,2 & 4,94 & 5,5 \\
C2 & 4,4 & 1,85 & 4 \\
C3 & 39 & 13,48 & 38 \\
C4 & 8,5 & 5,19 & 7 \\
C5 & 0 & 0 & 0 \\
C6 & 60,13 & 35,50 & 34 \\
C7 & 0 & 0 & 0 \\
C8 & 69,3 & 34,55 & 45 \\
C9 & 0 & 0 & 0 \\
Total & 188,53 & 62,89 & 173,5 \\
\hline
\end{tabular}


This evaluation is particularly important because is one of our main intentions to provide a template that permits fast configurations (maintaining the quality and satisfaction). We need to be aware that there are problems much more complex than others which could result in longer configurations. However it is our intention to define a template that can be configured in 5 minutes maximum. The Table 6 shows all the configuration times: average, standard deviation and the median for each point and the entire configuration.

Survey: post-test. This part had five statements and was conducted after the usability tests in order to collect users overall opinion of the experience?. In order to evaluate the level of agreement with each statement we used the Likert scale [13]. The Fig. 6 demonstrates the survey. All the decision-makers filled the survey without difficulty. However, during the post-test the evaluation of the statement number 5 created some discussion between the user and the evaluator in the majority of the cases. The evaluator needed to explain better what we intended to mean with "I felt limited by the template. There is more information that should be configured".

\section{S1: I felt satisfied with the problem's configuration easiness.}

Strongly Agree $\square \quad$ Agree $\square \quad$ Undecided $\square \quad$ Disagree $\square$ Strongly Disagree $\square$
S2: I felt satisfied with the problem's configuration speed.
Strongly Agree $\square \quad$ Agree $\square \quad$ Undecided $\square \quad$ Disagree $\square \quad$ Strongly Disagree $\square$
S3: If I had to use this template again I would feel more prepared.
Strongly Agree $\square \quad$ Agree $\square \quad$ Undecided $\square \quad$ Disagree $\square \quad$ Strongly Disagree $\square$
S4: This template was boring to use.
Strongly Agree $\square \quad$ Agree $\square \quad$ Undecided $\square \quad$ Disagree $\square \quad$ Strongly Disagree $\square$

S5: I felt limited by the template. There is more information that should be configured.

Strongly Agree $\square \quad$ Agree $\square \quad$ Undecided $\square \quad$ Disagree $\square \quad$ Strongly Disagree $\square$

Fig. 6. Survey: post-test

The Table 7 presents the surveys' results.

Table 7. Survey results

\begin{tabular}{lllllll}
\hline Statement & $\begin{array}{c}\text { Strongly } \\
\text { Agree }\end{array}$ & Agree & Undecided & Disagree & $\begin{array}{c}\text { Strongly } \\
\text { Disagree }\end{array}$ & Total \\
\hline S1 & 3 & 19 & 7 & 1 & 0 & 30 \\
S2 & 8 & 18 & 4 & 0 & 0 & 30 \\
S3 & 13 & 17 & 0 & 0 & 0 & 30 \\
S4 & 0 & 0 & 0 & 26 & 4 & 30 \\
S5 & 0 & 3 & 17 & 7 & 3 & 30 \\
\hline
\end{tabular}




\section{Discussion}

Reading the literature it is easy to understand the necessity of a good standard to define templates for multi-criteria problems. Is not the intention of this work to discourse about all the problems behind the UbiGDSS. However, it seems clear how the most complex negotiation model, with the best theoretical results can be a failure if some important points are not solved. When we work in a field such as the group decision-making it is fundamental to be aware that the final product will be used by human-beings, so it is important to design products according to their needs. Considering we are focused in UbiGDSS, it is clear that the end users will be very busy people. So, we should be especially careful with models that require a lot of information to work with and this is only possible if the information is configured without mistakes. As an example, let us consider a questionnaire with an average response time of 15 minutes. This questionnaire intends to understand well the decision-maker. Besides this questionnaire, the decision-maker also has to configure the problem itself. Looking at the kind of users that would use this type of product, maybe they will not be patient enough to answer to everything and more important than that, such a lengthy questionnaire could lead to them start answering randomly just with the purpose to end it. This action can result in several problems: (1) decision-maker does not want to use the system again; (2) the negotiation model will not work because the information is incorrect, etc.

Previously in this document we stated some factors that we consider to be important to the success of an UbiGDSS: non-mandatory configurations, usability, problem data always accessible, allow the decision-maker to model its opinion truthfully, and be complex enough whenever it is necessary. So, we defined our general templates based in these premises.

We believe that with practice the users will get even better results than those obtained in our case study. However, our case study had very good results in general. Regarding to the easiness to use our template the results were perfect, none user got lost during the configuration, everyone configured the problem in the correct order, they did not do mistakes and everyone used the correct "info" buttons. The configuration times of each point were quite good. In addition, our expectation for the configuration not requiring more than 5 minutes to be completed was verified with the average configuration time of 188,53 seconds (a bit more than 3 minutes). The user who used more time to configure the problem took almost 6 minutes, the second highest took 310 seconds and the third highest took 298 seconds (almost 5 minutes). Only 2 users required more than 5 minutes $(6,66 \%)$. The points which required more time to be configured were: conflict style, alternatives evaluation and attributes evaluation. The "conflict styles" was also the point with more clicks in the respective "info" button, which makes sense considering that no user was familiar with this terminology and because of that had the necessity to learn about it. In our opinion, if they perform another case study using the same template the time to configure "conflict styles" will decrease exponentially. Every user clicked in the "conflict styles" "info" button and some of them more than 1 time. Almost all of the users clicked in the "info" button of the alternatives and attributes evaluation. One important thing that was observed was 
that every user consulted the problem data while they were configuring the alternatives and criteria.

In respect to the survey (post-test), 73,33\% of the users considered the template an easy way to configure a problem like this, $23,33 \%$ were undecided and only one did not considered the template easy to use. Relatively to how fast is to configure a multicriteria problem using our template, the big majority $(86,66 \%)$ felt satisfied with the configuration speed and only 4 were undecided. $100 \%$ of the users considered that they will be more prepared to use this template if they had to repeat the experience. Nobody considered this template boring, which is a great result for us and finally $56,66 \%$ of the users were undecided regarding S5. In our opinion most of the users who answered "undecided" relatively to the S5 did so because we think that only if they used a template like this in a real system, in real world, during a considerable period of time then they would be more prepared to correctly formulate an opinion to answer to S5. In general our template achieved good results, the majority of the users felt satisfied with the easiness and the configuration speed, nobody felt bored using our template and the majority agreed they will feel more prepared if they have to use the template again.

Apart of the case study, our template includes a configuration that provides a lot of data to be used in UbiGDSS and in the negotiation models nowadays and the techniques used to get the data permits to deduce and understand even more information. For instance, the alternatives and attributes configuration are performed according to an unconscious comparison.

\section{Conclusions and Future Work}

Defining a balance between the amount of information that needs to be configured by the decision-maker and at the same time assure usability, quickness and perceptibility is not an easy task. However, it is important to notice that due to its context, if this task is not properly executed, it can affect the system's viability. The work presented in this paper is a result of several years of study in the area of Group Decision Support Systems and even if we consider that there is a lot of brilliant work in this area, there are still problems that need to be addressed that we think that may be responsible to destroy what could be the success of UbiGDSS.

This work had the main goal to propose a general template which will allow to obtain, infer and create intelligence about "every" question that affects the group decision-making process. Our proposal is divided in three sections: Problem Data, Personal Configuration and Problem Configuration. Furthermore, we proposed a specification of our general template as an example that shows how these points could be implemented, and at the same time assure the usability and simplicity of the configuration for any kind of electronic device. In order to validate our proposal, we conducted a case study where a group of decision-makers configured a simulated problem and in the end filled a survey. Our approach yielded excellent results, demonstrating high levels of usability and configuration speed. Besides, the decision-makers in general, 
considered the template very perceptible and that satisfies their needs in terms of amount of information they consider necessary for the decision process.

As future work, we want to establish in literature a reference standard for "how to define a multi-criteria problem template for UbiGDSS". We intend to continue studying this issue in terms of usability and understand which other data decision-makers consider important to collect in this context. Besides, we also intend to perform a new case study where the decision-makers need to fill out more than one problem because we believe that with some experience they will to fill faster fundamentally the section of personal configuration.

\section{Acknowledgements}

This paper is a revised and an expanded version of a paper entitle "Individual Definition of Multi-Criteria Problems in Ubiquitous GDSS" presented at International Conference Ubiquitous Computing and Multimedia Applications 2015, Porto, Portugal [14]. This work is part-funded by ERDF - European Regional Development Fund through the COMPETE Programme (operational programme for competitiveness) within project FCOMP-01-0124-FEDER-028980 (PTDC/EEISII/1386/2012) and by National Funds through the FCT - Fundação para a Ciência e a Tecnologia (Portuguese Foundation for Science and Technology) with the João Carneiro PhD grant with the reference SFRH/BD/89697/2012.

\section{References}

1. Carneiro, J., Santos, R., Marreiros, G., Novais, P.: Overcoming the Lack of HumanInteraction in Ubiquitous Group Decision Support Systems. (2014)

2. Kwon, O., Yoo, K., Suh, E.: UbiDSS: a proactive intelligent decision support system as an expert system deploying ubiquitous computing technologies. Expert systems with applications 28, 149-161 (2005)

3. Marreiros, G., Ramos, C., Neves, J.: Dealing with emotional factors in agent based ubiquitous group decision. In: Embedded and Ubiquitous Computing-EUC 2005 Workshops, pp. 41-50. Springer, (2005)

4. Carneiro, J., Laranjeira, J., Marreiros, G., Freitas, C., Santos, R.: A Context-aware Model to Support Ubiquitous Group Decision Making. Journal of Internet Services and Information Security (JISIS) 2, 105-118 (2012)

5. Carneiro, J., Marreiros, G., Novais, P.: An Approach for a Negotiation Model Inspired on Social Networks. In: PAAMS. (2015)

6. Carneiro, J., Santos, R., Marreiros, G., Novais, P.: UbiGDSS: A Theoretical Model to Predict Decision-Makers' Satisfaction. International Journal of Multimedia and Ubiquitous Engineering 10, 191-200 (2015)

7. Carneiro, J., Santos, R., Marreiros, G., Novais, P.: Understanding Decision Quality through Satisfaction. Highlights of Practical Applications of Heterogeneous Multi-Agent Systems. The PAAMS Collection, pp. 368-377. Springer (2014)

8. Carneiro, J., Marreiros, G., Novais, P.: Using Satisfaction Analysis to Predict Decision Quality. International Journal of Artificial Intelligence ${ }^{\mathrm{TM}} 13,45-57$ (2015) 
9. Flanagin, A.J., Metzger, M.J.: Digital media and youth: Unparalleled opportunity and unprecedented responsibility. Digital media, youth, and credibility 5-27 (2008)

10. Martinho, D., Carneiro, J., Marreiros, G., Novais, P.: Dealing with Agents' Behaviour in the Decision-Making Process. In: SOOW. (2015)

11. Price, D.D., McGrath, P.A., Rafii, A., Buckingham, B.: The validation of visual analogue scales as ratio scale measures for chronic and experimental pain. Pain 17, 45-56 (1983)

12. Preece, J.R., Sharp, Y.: H.: Interaction Design: Beyond human computer interaction. USA: John Wiley \& Sons, Inc. (2002)

13. Likert, R.: A technique for the measurement of attitudes. Archives of psychology (1932)

14. Carneiro, J., Martinho, D., Marreiros, G., Novais, P.: Individual Definition of MultiCriteria Problems in Ubiquitous GDSS. (2015) 